Case Report

\title{
Report of a Sporadic Caudal Regression syndrome with Cleft Lip and Palate
}

\author{
Rathika D. Shenoy ${ }^{1}$, Swathi Sunil Rao ${ }^{2}$, Vijaya D. Shenoy ${ }^{3}$, Vikram Shetty ${ }^{4}$ \\ ${ }^{1}$ Professor, ${ }^{2}$ Assistant Professor, ${ }^{3}$ Professor \& Head, Department of Paediatrics, K.S. Hegde Medical Academy, Nitte \\ University, Deralakatte, Mangalore, ${ }^{4}$ Director, Department of Paediatrics, Nitte Meenakshi Institute of Craniofacial \\ Surgery, KS Hegde M edical Academy, Nitte University, Deralakatte, M angalore - 575 018, Karnataka, India.
}

*Corresponding Author: Swathi Sunil Rao, \#3/77/1A1, Kanasu, Someshwara, Mangalore-575022, Karnataka, India.

Mobile: +9190085 66112, E-mail : swathiyrao@yahoo.com.

$\begin{array}{ll}\text { Received } & : 24-02-2016 \\ \text { Review Completed } & : \text { 01-08-2016 } \\ \text { Accepted } & : 05-08-2016\end{array}$

Keywords : Sacral agenesis; tethered cord; unilateral renal agenesis

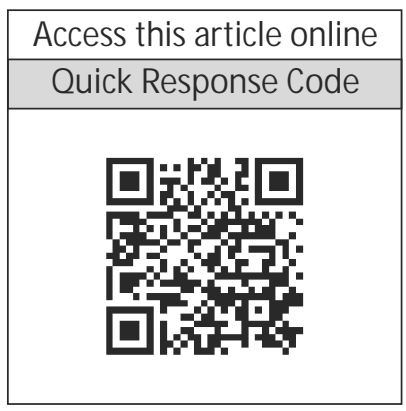

\begin{abstract}
:
Caudal regression syndrome is a rare congenital malformation and can be associated with lower limb and renal anomalies. However caudal regression syndrome and cleft lip and palate (CLP) rarely occur together, the prevalence of sacral anomalies in children with CLP being $0.1 \%$. We hereby report an eighteen month old male child with sporadic lumbosacral agenesis type III with tethered cord, hypospadias, unilateral renal agenesis and CLP for its rarity.
\end{abstract}

\section{Introduction}

Caudal regression syndrome (CRS) (OM IM 600145) also referred to as caudal dysgenesis is a rare congenital malformation with an incidence of 1 to 2.5 per 100,000 pregnancies. ${ }^{1}$ It is characterized by variable absence of caudal spine and sacrum with involvement of spinal cord, urogenital system, and lower limbs. It is presently localized to VANGL1 gene on chromosome 1p13. Cleft lip and palate (CLP) are one of the commonest birth defects with a prevalence of 1 in 1000 live births and in one-third are associated with other congenital anomalies. ${ }^{2}$ The prevalence of sacral anomalies with CLP and other noncleft defects are reportedly 0.1 and $1 \%$ respectively. ${ }^{1}$ We hereby report a young child with lumbosacral agenesis type III with tethered cord, hypospadias, unilateral renal agenesis and CLP for its rarity.

\section{Case History}

A male child was first seen by us at 18 months with orofacial defect, lower limb deformity, urinary and faecal incontinence since birth. He was the only child of healthy, young, non-consanguineous parents. There was no maternal diabetes during antenatal period. He was born at term with birth weight of $2500 \mathrm{~g}$ by caesarean section due to breech presentation. His weight and head circumference were normal between third to fifteenth centile. There was no dysmorphism to suggest a syndromic CLP. Neurological examination showed involvement of lower lumbar and sacral nerve fibres especially on left. The left lower extremity was wasted and short; fixed hyperextended knee (genu recurvatum) and rockerbottom foot were present (Figures 1B).The child had neurogenic bladder and patulous anal sphincter. Genital examination showed a pre-penile scrotum with bilateral 
palpable gonads, peno-scrotal hypospadias and severe chordee (Figure 1B). Upper limb examination was normal.

Radiograph showed lumbosacral agenesis (Figures $2 \mathrm{~A}$ and 2B). In addition, developmental dysplasia of left hip, genu recurvatum, vertical talus and talo-navicular dislocation were noted. Magnetic resonance imaging (MRI) of the spine showed tethered cord with hydromelia at L2-L3 level (Figure $3 \mathrm{~A}$ and $3 \mathrm{~B}$ ). The vertebrae below $\mathrm{L} 2$ level, sacrum and coccyx were not well delineated suggesting caudal regression syndrome. Neuroimaging of brain was normal. Ultrasound abdomen showed left renal agenesis. Renal functions and echocardiography were normal. Karyotype showed 46, XY. Fluorescent Insitu Hybridization (FISH) for 22q11 deletion was negative. Child was operated for cleft lip and palate. Partial laminectomy with de-tethering of cord was done and on follow-up at three years child was able to walk but with an abnormal gait. Parents did not consent for further evaluation of renal system or corrective surgery for hypospadias.

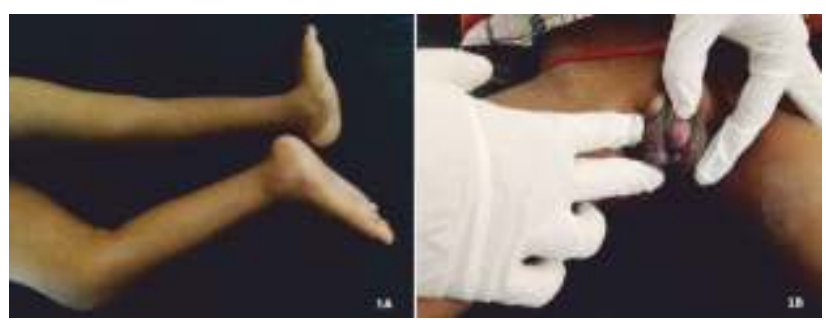

Figure 1 : 1A. Wasted left lower limb with genu recurvatum and rocker bottom foot, 1B : Prepenile scrotum with penoscrotal hypospadias and chordee.

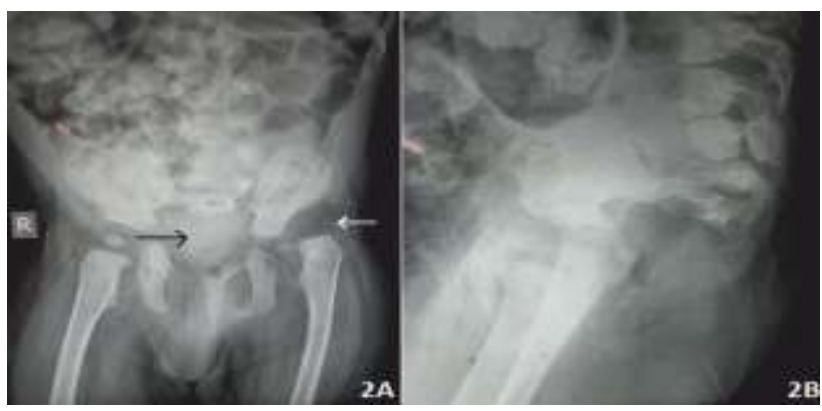

Figure 2 : 2A. Radiograph of the pelvis anteroposterior view showing agenesis of the sacrum (black arrow), Developmental dysplasia of left hip (white arrow), 2B: Lateral radiograph of the pelvis showing agenesis of sacrum and lower lumbar vertebrae.

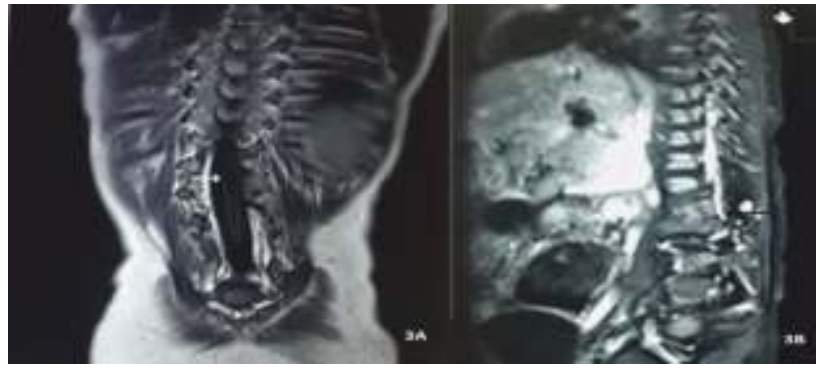

Figure 3 : 3A. Low lying spinal cord (L5 level) with hydromelia (Arrow), 3B. Low lying spinal cord with tethering at L2-L3 level (Arrow)

\section{Discussion}

CRS has been classified by Renshaw (1978) into four types with the degree of severity increasing from type I through IV. ${ }^{3}$ Type I is total or partial unilateral sacral agenesis and is seen in along with pre-sacral mass and anal stenosis as Currarino triad. Typell, the most common, istotal or partial bilateral symmetric sacral agenesis where the cord is lowlying and tethered. In the last two types, there is total sacro-coccygeal with variable lumbar agenesis with the last lumbar segment articulating with the ilia in type III or resting on the fused ilia in type IV. Types II and III usually have stable spino-pelvic junctions. Type IV often has progressive spinopelvic kyphosis and is associated with severe lower limb anomalies. Our case best fitted into type III which is the rarest variety. The breech presentation and the severe asymmetric limb deformity suggested fetal hypokinesia secondary to spinal cord malformation inutero.

About $25 \%$ of CRS is associated with maternal diabetes mellitus. ${ }^{5}$ Drug-related etiology has also been implicated. M ost of the previous studies strongly suggest that various chemicals may play a teratogenic role in the genesis of CRS. ${ }^{5}$ Genital and renal anomalies seen in the index case with lumbo-sacral agenesis and tethered cord are reported also with VACTERL association and velo cardio facial syndrome (VCFS) which is part of 22q11 deletion. ${ }^{4}$ VACTERL and CRS can have overlapping features which are reported in previous studies. ${ }^{6,7}$ However the propositus did not qualify for VACTERL since atleast three components are necessary and the core features namely trachea-esophageal fistula or anorectal malformation were absent. CLP, unilateral renal 
agenesis and hypospadias may be seen in VCFS; sacral agenesis is also reported. However our patient did not have cardiac defects, hypocalcemia or immune deficiency consistently seen with this syndrome and FISH for 22q11 was negative.

\section{References:}

1. Mia M A H, Hoque M, Malik M A, Akhter K R, Hossain M Z. Caudal regression syndrome in the infant of a diabetic mother: $A$ case report.DS(Child) HJ .2012: 28(1);46-48.

2. Rittler M, Camelo J S L, Castilla E E, Bermejo E, Cocchi G et al. Preferential Associations Between Oral Clefts and Other Major Congenital Anomalies. Cleft Palate-Craniofacial Journal.2008;5:525532.

3. Renshaw TS. Sacral Agenesis. The Pediatric Spine - Principles and Practice. New York: Raven Press; 1994:2214.

4. Solomon BD.VACTERL/VATER Association. Orphanet Journal of Rare Diseases. 2011;6:56.

5. Yeniel AO, Ergenoglu AL, Sagol S. Caudal regression- a rare case. J Turkish-German Gynecol Assoc. 2011;12:186-188.

6. Online Mendelian Inheritance in Man, OM IM (TM).Johns Hopkins University, Baltimore, MD. MIM Number: 192430: 03/21/2014: Available at http:// www.omim.org/

7. ljaz L, Shiekh A. Overlapping features of Caudal regression syndrome and VACTERAL complexinaneonate. APSJ Case Rep.2010;1:10.
In the absence of any risk factors contributing to etiology, the combination of these two multifactorial disorders in the indexcase may be considered to be sporadic. 